Imperial/TP/96-97/36

hep-th/9703091

March 1997

\title{
Quantum Group Generators in Conformal Field Theory
}

\author{
Jens SCHNITTGER円 \\ Blackett Laboratory, Theory Group, Imperial College, \\ London SW7 2BZ, United Kingdom \\ and \\ CNRS-Lab. de Mathématiques et Physique Théorique (UPRES A 6083), \\ Université de Tours, Parc Grandmont, F-37200 Tours, France
}

\begin{abstract}
Two approaches to the construction of symmetry generators for the quantum group $U_{q}(l(2))$ in conformal field theory are presented, in the concrete context of $2 \mathrm{~d}$ gravity. The first works with an extension of the physical phase space and has been successfully applied already to WZW theory. We show that the result can be used also for Liouville theory and related models by employing Hamiltonian reduction. The second is based on a completely new idea and realizes the quantum group symmetry intrinsically, on the physical phase space alone.
\end{abstract}

\footnotetext{
${ }^{1}$ supported in part by grant ERBCHBICT941380
} 


\section{Introduction}

Quantum Groups are known to govern the structure of many integrable systems, such as Sine-Gordon theory or the XXZ chain, and in particular of conformal field theory (CFT), where prominent examples are Liouville/Toda theory, minimal models, or WZW theory. The quantum group determines both the operator products and the exchange (braiding) relations of the chiral operator algebra. The nonchiral observables can be viewed as singlets of the quantum group. Thus the quantum group acts naturally in an extended phase space $\Gamma_{L} \times \Gamma_{R}$ of left- and rightmoving degrees of freedom. This is loosely analogous to the case of gauge theories, where observables by definition are singlets under the gauge group as well, and the extended phase space is provided by the gauge fields. The rôle of a specified gauge for the gauge fields is played by the monodromy of the chiral vertex operators. In the example of WZW theory, the general solution $g\left(x^{+}, x^{-}\right)$with periodic boundary conditions is

$$
g\left(x^{+}, x^{-}\right)=g_{L}\left(x^{+}\right) g_{R}^{-1}\left(x^{-}\right)
$$

in light-cone coordinates $x^{ \pm}=x \pm t$. Here, $g_{L}$ and $g_{R}$ are periodic up to the monodromy matrix $\gamma$,

$$
\begin{gathered}
g_{L}\left(x^{+}+2 \pi\right)=g_{L}\left(x^{+}\right) \gamma \\
g_{R}\left(x^{-}+2 \pi\right)=g_{R}\left(x^{-}\right) \gamma .
\end{gathered}
$$

Thus, the monodromy matrix $\gamma$ specifies the "gauge", and gauge transformations $g_{L} \rightarrow g_{L} g_{0}, g_{R} \rightarrow g_{R} g_{0}\left(\Rightarrow \gamma \rightarrow g_{0}^{-1} \gamma g_{0}\right)$ do not change the "observable" $g\left(x^{+}, x^{-}\right)$. The physical phase space is therefore given by the subset of $\Gamma_{L} \times \Gamma_{R}$ with equal monodromies $\left(\gamma_{L}=\gamma_{R}\right)$, divided by the set of gauge transformations $g_{0}$ :

$$
\Gamma_{\text {phys }}=\Gamma_{L} \times\left.\Gamma_{R}\right|_{\left(\gamma_{L}=\gamma_{R}\right)} / G
$$

where $G$ is the gauge group. The above relation has meaning a priori only when $G$ is a classical group, and we are considering the classical phase space. The q-deformation of the classical symmetry and the quantization of the system need not be correlated[; however, in the case we will consider concretely, that of Liouville theory, the two deformations are identified[? The

\footnotetext{
2 This is why the name "quantum group" is in general misleading.

${ }^{3}$ The situation is somewhat more complicated in the WZW case [1].
} 
way in which relation Eq. (2) carries over to the quantum case is then that quantum observables are formed from left- and rightmoving vertex operators with equal monodromies in such a way that they are invariant under the (suitably defined) action of the universal enveloping algebra, $U_{q}(s l(2))$ in the case of Liouville theory. It is important to note that the monodromy matrix is dynamical; that is, its eigenvalues are functionals on the physical phase space, as is explicit in the WZW example above.

\section{Covariant chiral vertex operators for $\mathrm{U}_{\mathrm{q}}(\mathrm{sl}(2))$}

In the work of refs. [2] and [3] it was revealed that $2 \mathrm{~d}$ gravity/Liouville theory naturally provides a set of chiral vertex operators $\xi\left(x^{+}\right), \bar{\xi}\left(x^{-}\right)$with a fixed triangular monodromy, which transform covariantly under $U_{q}(s l(2))$. They thus form spin $J$ representations $\xi_{M}^{(J)}, \bar{\xi}_{M}^{(J)}$. Accordingly, the product of chiral vertex operators behaves like the product of representations; in particular the interchange of two representations is given by the exchange matrix (or $R$ matrix):

$$
\xi_{M_{1}}^{\left(J_{1}\right)}\left(x_{1}^{+}\right) \xi_{M_{2}}^{\left(J_{2}\right)}\left(x_{2}^{+}\right)=\left(J_{1}, J_{2}\right)_{M_{1} M_{2}}^{M_{2}^{\prime} M_{1}^{\prime}} \xi_{M_{2}^{\prime}}^{\left(J_{2}\right)}\left(x_{2}^{+}\right) \xi_{M_{1}^{\prime}}^{\left(J_{1}\right)}\left(x_{1}^{+}\right)
$$

where

$$
\left(J, J^{\prime}\right)_{M M^{\prime}}^{N^{\prime} N}=\left\langle J, M\left|\otimes\left\langle J^{\prime}, M^{\prime}|\mathbf{R}| J, N\right\rangle \otimes\right| J^{\prime}, N^{\prime}\right\rangle
$$

and

$$
\mathbf{R}=e^{\left(-2 i h J_{3} \otimes J_{3}\right)}\left(1+\sum_{n=1}^{\infty} \frac{\left(1-e^{2 i h}\right)^{n} e^{i h n(n-1) / 2}}{\lfloor n\rfloor !} e^{-i h n J_{3}}\left(J_{+}\right)^{n} \otimes e^{i h n J_{3}}\left(J_{-}\right)^{n}\right) .
$$

is the universal $R$-matrix of $U_{q}(\operatorname{sl}(2)) .\left(\lfloor x\rfloor:=\frac{q^{x}-q^{-x}}{q-q^{-1}}\right.$ denotes quantum numbers, as usual.) The relation written is valid for $x_{1}^{+}>x_{2}^{+}$, and the inverse exchange matrix, with $J_{1}$ and $J_{2}$ exchanged, is relevant in the other case. We normally consider all $\xi_{M}^{(J)}$ operators on the interval $x \in[0,2 \pi]$, though they are defined for arbitrary $x$. The connection between different periodicity intervals is given by the monodromy operation (cf. section 6). Relation Eq.(3) is invariant under the transformation $\xi_{M}^{(J)} \rightarrow T_{M N} \xi_{N}^{(J)}$ with $\left[T_{M_{1} M_{2}}^{(J)}, \xi_{M}^{(J)}\right]=0$, provided $T$ fulfills the famous Faddeev-Reshetikhin-Takhtadzhian relation [1]

$$
T^{1} T^{2} R=R T^{2} T^{1}
$$


On the lefthand side, $T^{1}$ acts on the first lower index of $R$, and similarly for the rest. The matrix elements of $T$ can be viewed as elements of $F_{q}(S L(2))=U_{q}^{*}(s l(2))$, the quantized algebra of functions on the group. Their noncommutativity reflects a crucial property of the quantum group symmetry: It is a symmetry of the Poisson-Lie type. Classically, this means that the Poisson structure of the theory is invariant under the symmetry only if the symmetry group is equipped with a nontrivial Poisson structure itself [5], namely the classical limit of Eq.(4) which is given in terms of the classical $r$-matrix $R=1+i h r+\mathcal{O}\left(h^{2}\right)$.

\section{Quantum Group invariants}

From the $\xi_{M}^{(J)}, \bar{\xi}_{M}^{(J)}$, it is easy to form singlets under the quantum group. In Liouville theory, they represent the observables $e^{-J \alpha_{-} \Phi}$, the exponentials of the Liouville field [6] ( $\alpha_{-}$is the semiclassical screening charge of the Coulomb gas):

$$
e^{-J \alpha_{-} \Phi}=\sum_{M}(-1)^{J+M} q^{J-M} \xi_{M}^{(J)}\left(x^{+}\right) \bar{\xi}_{-M}^{(J)}\left(x^{-}\right)
$$

The same formula can be reinterpreted as providing local observables in general Dotsenko-Fateev type models built from integer powers of screenings in the Coulomb gas picture (without restriction on the central charge) and, by taking a limit to rational values of the central charge, in minimal models [7]. The transformation $\xi_{M}^{(J)} \rightarrow T_{M N} \xi_{N}^{(J)}$ of section 2 - the quantum PoissonLie map - is a map from $F_{q}(\Gamma)$ to $F_{q}(\Gamma) \otimes F_{q}(S L(2))$, where $F_{q}(\Gamma)$ denotes the quantized functions on the phase space. By dualization, this can be turned into an action of the algebra $U_{q}(s l(2))$ on $F_{q}(\Gamma)$ : For any element $a_{q} \in U_{q}(s l(2))$, we have

$$
a_{q} \triangleright \xi_{M}^{(J)}=<a_{q}, T_{M N} \xi_{N}^{(J)}>
$$

where $<,>$ is the canonical pairing between $U_{q}(s l(2))$ and $F_{q}(S L(2))$. For $a_{q}=J_{ \pm}, J_{3}$ this gives back the usual action of the $U_{q}(\operatorname{sl}(2))$ generators on a representation of spin $J$ :

$$
J_{ \pm}|J M>=\sqrt{\lfloor J \mp M\rfloor\lfloor J \pm M+1\rfloor}| J M \pm 1>,
$$




$$
J_{3}|J M>=M| J M>.
$$

Moreover, on a product of representations, the algebra acts by the coproduct. We take it to be given by

$$
\Delta\left(J_{ \pm}\right)=J_{ \pm} \otimes q^{J_{3}}+q^{-J_{3}} \otimes J_{ \pm}, \quad \Delta\left(J_{3}\right)=J_{3} \otimes 1+1 \otimes J_{3} .
$$

For the product of a leftmoving and a right-moving representation - which differ essentially by complex conjugation - one should use the above coproduct with $q$ replaced by $q^{-1}[7]$. It is then immediate to check that $e^{-J \alpha_{-} \Phi}$ is indeed invariant under this action.

\section{Poisson-Lie generators}

In the previous section, we have introduced the action of the quantum group "by hand", that is, by direct linear action. On the other hand, the gauge theory analogy lets us expect that the theory should actually furnish Hamiltonian generators - the Noether charges - which generate the symmetry by Poisson brackets (or commutators in the quantum case):

$$
\delta f=\{Q, f\}
$$

where $f$ is any function on the phase space. However, as we have already remarked in section 2, the symmetry we consider here is of the PoissonLie type. This means that already on the classical level, the action of the generators is modified. The appropriate generalization of Eq.(6) is [8]

$$
\delta_{a} f=<a,\{m, f\} m^{-1}>
$$

Here $m$ is an element of the dual group $G^{*}$, the socalled moment map, while $a$ is an element of the algebra $A$ of $G$, and $\langle$,$\rangle is the canonical pairing$ between elements of $A$ and $A^{*}$. On the quantum level, the Poisson-Lie action is characterized by commutation relations of the form

$$
M\left(a_{q}\right) \mathcal{O}=\sum\left(a_{q}^{(1)} \triangleright \mathcal{O}\right) M\left(a_{q}^{(2)}\right)
$$

where $\Delta\left(a_{q}\right)=\sum a_{q}^{(1)} \otimes a_{q}^{(2)}$ is the coproduct of an element of the quantum universal enveloping algebra, and $\mathcal{O}$ is an element of the quantized functions 
on the phase space, i.e., an operator. Furthermore, $M\left(a_{q}\right)$ is a homomorphism from the universal enveloping algebra to the quantized functions on the phase space, the (dualized) quantum moment map. The action of $a_{q}^{(1)}$ on $\mathcal{O}$ is just the linear action discussed above, so for the case at hand we obtain

$$
M\left(J_{i}\right) \xi_{M}^{(J)}=\xi_{N}^{(J)}\left(J_{i}^{(1)}\right)_{N M} M\left(J_{i}^{(2)}\right)
$$

Before we go into the construction of $m$ and its quantum generalization, it is important to note the following point: Any moment map $m$ capable of generating $s l(2))$ transformations of the fields $\xi_{M}^{(J)}, \bar{\xi}_{M}^{(J)}$ must necessarily be defined on an extended phase space $\Gamma_{\text {ext }} \supset \Gamma_{\text {phys }}$. This is because, for the $\xi_{M}^{(J)}, \bar{\xi}_{M}^{(J)}$ of Eq.(3) with their fixed monodromy, the correspondence between $e^{-J \alpha_{-} \Phi}$ and $\xi_{M}^{(J)}, \bar{\xi}_{M}^{(J)}$ is one-to-one. Thus, $\xi_{M}^{(J)}$ and $\bar{\xi}_{M}^{(J)}$ can be viewed as functions on $\Gamma_{\text {phys }}$, and therefore must be invariant under the symmetry! The natural way out of this problem is to formulate $m$ on the extended phase space $\Gamma_{L} \times \Gamma_{R}$ instead of $\Gamma_{\text {phys }}$, so that also the eigenvectors, and not just the eigenvalues, of the monodromy matrix are considered as dynamical. We will work out in section 5 on the classical level that indeed in this way one can obtain the desired Poisson-Lie action, by Hamiltonian reduction from the WZW theory.

Of course, passing to $\Gamma_{\text {ext }} \supset \Gamma_{\text {phys }}$ introduces a redundancy, just as working with dynamical gauge fields does in gauge theory. Surprisingly, there exists a possibility to avoid this redundancy, while still achieving the essential part of our goal. The idea is to work with generators that act nontrivially on $\xi_{M}^{(J)}, \bar{\xi}_{M}^{(J)}$ - hence do not leave $\Gamma_{\text {phys }}$ invariant globally - but preserve the invariance of a subset of observables, i.e. functions on $\Gamma_{\text {phys }}$. This approach is in fact suggested by a simple observation on the structure of the $R$ - matrix, and will be carried out directly on the quantum level in section 6 .

\section{Classical moment map for WZW and Liou- ville theory in extended phase space}




\subsection{The case of SL(2) WZW}

The moment map for the WZW model was given in ref. [5]. We recapitulate very briefly the main statements for the case of $S L(2)$, partially in order to prepare the Hamiltonian reduction to Liouville theory. Let us write the left-moving WZW group element on $\Gamma_{L}$ as

$$
g_{L}\left(x^{+}\right)=\left(\begin{array}{cc}
1 & v_{L}\left(x^{+}\right) \\
0 & 1
\end{array}\right)\left(\begin{array}{cc}
1 & 0 \\
w_{L}\left(x^{+}\right) & 1
\end{array}\right)\left(\begin{array}{cc}
e^{\Phi_{L}\left(x^{+}\right)} & 0 \\
0 & e^{-\Phi_{L}\left(x^{+}\right)}
\end{array}\right) g_{0 L}
$$

where $v_{L}$ and $w_{L}$ are periodic, while $\Phi_{L}\left(x^{+}+2 \pi\right)=\Phi_{L}\left(x^{+}\right)+2 \pi p_{L}$. Furthermore, $g_{0 L}$ is a constant matrix describing the eigenvectors of the monodromy matrix $\gamma_{L}$ of Eq.(运),

$$
\gamma_{L}=g_{0 L}^{-1} e^{2 \pi \tau_{L}} g_{0 L}, \quad \tau_{L}=\left(\begin{array}{cc}
p_{L} & 0 \\
0 & -p_{L}
\end{array}\right)
$$

Similarly, we writef

$$
g_{R}\left(x^{-}\right)=\left(\begin{array}{cc}
1 & 0 \\
w_{R}\left(x^{-}\right) & 1
\end{array}\right)\left(\begin{array}{cc}
1 & v_{R}\left(x^{-}\right) \\
0 & 1
\end{array}\right)\left(\begin{array}{cc}
e^{\Phi_{R}\left(x^{-}\right)} & 0 \\
0 & e^{-\Phi_{R}\left(x^{-}\right)}
\end{array}\right) g_{0 R}
$$

with the corresponding properties of $p_{R}$ and $g_{0 R}$. On the physical phase space $\Gamma_{\text {phys }}$ we have $g_{0 L}=g_{0 R}$ and $p_{L}=p_{R}$. The symplectic form $\Omega$ can be written $\Omega=\Omega_{L}-\Omega_{R}$, with

$$
\begin{gathered}
\Omega_{L}\left(g_{L}\right)=\frac{k}{4 \pi} \int_{0}^{2 \pi} d x^{+} \operatorname{tr}\left[\left(g_{L}^{-1} d g_{L}\right) \wedge \partial_{+}\left(g_{L}^{-1} d g_{L}\right)\right] \\
+\frac{k}{4 \pi} \operatorname{tr}\left[g_{L}^{-1} d g_{L}(0) \wedge d \gamma_{L} \gamma_{L}^{-1}\right]-\frac{k}{4 \pi} \rho\left(\gamma_{L}\right)
\end{gathered}
$$

and the same with $g_{L} \rightarrow g_{R}$ for $\Omega_{R}$. The two-form $\rho(\gamma)$ is a priori arbitrary as it drops out of $\Omega$. However, it is possible to choose $\rho$ such that $d \Omega_{L}=0$ and $d \Omega_{R}=0$ separately on a dense open subset of the respective phase spaces $\Gamma_{L}, \Gamma_{R}$. Following ref. [5], we take

$$
\rho(\gamma)=\operatorname{tr}\left\{\left(\gamma^{-}\right)^{-1} d \gamma^{-} \wedge\left(\gamma^{+}\right)^{-1} d \gamma^{+}\right\}
$$

\footnotetext{
${ }^{4}$ In the following, we will mainly concentrate on the left-movers, as the story for the right-movers is very similar.
} 
Here $\gamma=\gamma^{-}\left(\gamma^{+}\right)^{-1}$ is the triangular decomposition of $\gamma$, i.e.

$$
\gamma^{+}=\left(\begin{array}{cc}
\kappa & * \\
0 & 1 / \kappa
\end{array}\right), \gamma^{-}=\left(\begin{array}{cc}
1 / \kappa & 0 \\
* & \kappa
\end{array}\right)
$$

The structure of $\Omega_{L, R}$ admits a PL symmetry $g_{l} \rightarrow g_{L} g, g_{R} \rightarrow g_{R} g(g$ a constant matrix), provided we define on $F(G)$ the Poisson structure

$$
\left\{g_{1}, g_{2}\right\}:=\left[g_{1} g_{2}, r\right]
$$

Here, $g_{1}:=g \otimes 1, g_{2}:=1 \otimes g$, and $r=\frac{\pi}{k}\left(e_{+} \otimes e_{-}-e_{-} \otimes e_{+}\right)$with $e_{ \pm}$ the raising/lowering generators of $\operatorname{sl}(2)$. Furthermore, $r$ is related to the solutions $r^{ \pm}$of the classical Yang-Baxter equation for $\operatorname{sl}(2)$ via

$$
r^{ \pm}=r \pm \frac{2 \pi}{k} C, C=\sum_{a} t^{a} \otimes t^{a}
$$

being the quadratic Casimir.

The generator of this PL symmetry - the classical moment map - is just given by the components $\left(\gamma^{+}, \gamma^{-}\right) \in G^{+} \otimes G^{-} \subset G^{*}$ of the monodromy matrix. One can indeed deduce directly from the symplectic forms $\Omega_{L, R}$ that for $m_{L}=\left(\gamma_{L}^{+}, \gamma_{L}^{-}\right)$,

$$
<\epsilon,\left\{m_{L}, g_{L}\right\} m_{L}^{-1}>=-t r_{1}\left(\epsilon \otimes g_{L} r^{+}\right)+t r_{1}\left(\epsilon \otimes g_{L} r^{-}\right)=-\frac{4 \pi}{k} \sum_{a} \operatorname{tr}\left(t^{a} \epsilon\right) g_{L} t^{a}
$$

where $\epsilon$ is any element of $\operatorname{sl}(2)$. This means that $m_{L}$ (and similarly $m_{R}$ ) properly generates the linear action of the algebra on $g_{L}$,

$$
\delta g_{L}=g_{L} \epsilon_{a} t^{a}, \epsilon_{a}=\operatorname{tr}\left(t^{a} \epsilon\right)
$$

\subsection{Hamiltonian reduction to Liouville theory}

Using the above result, we can now proceed to obtain the moment map for Liouville by Hamiltonian reduction [9]. We will show that the form of $m$ remains unaffected by the reduction, so that the same moment map, given by the monodromy, can be used. The reduction procedure is defined by the constraints

$$
j_{L}^{+}=\mu^{+}, \quad j_{R}^{-}=\mu^{-}
$$


with

$$
\begin{aligned}
& j_{L}^{+}=\operatorname{tr}\left(e_{+} j_{L}\right), j_{L}=g_{L} \partial_{+} g_{L}^{-1} \\
& j_{R}^{-}=\operatorname{tr}\left(e_{-} j_{R}\right), j_{R}=g_{R} \partial_{-} g_{R}^{-1}
\end{aligned}
$$

Using the parametrizations Eq.(10), this becomes

$$
\partial_{+} w_{L}+2 \partial_{+} \Phi_{L} w_{L}=\mu^{+}, \partial_{-} v_{R}+2 \partial_{-} v_{R}=-\mu^{-}
$$

The Liouville field is recovered from the Cartan part of the Gauss decomposition of $g=g_{L} g_{R}^{-1}$,

$$
g=\left(\begin{array}{ll}
1 & x \\
0 & 1
\end{array}\right)\left(\begin{array}{cc}
e^{\varphi / 2} & 0 \\
0 & e^{-\varphi / 2}
\end{array}\right)\left(\begin{array}{ll}
1 & 0 \\
y & 0
\end{array}\right),
$$

or

$$
e^{\varphi}=\frac{e^{2 \Phi_{L}\left(x^{+}\right)} e^{2 \Phi_{R}\left(x^{-}\right)}}{\left(1-w_{L} e^{2 \Phi_{L}} v_{R} e^{-2 \Phi_{R}}\right)^{2}}
$$

(note that $g_{0}$ drops out). Eq.(12) implies

$$
\begin{gathered}
w_{L}\left(x^{+}\right)=\mu^{+} e^{-2 \Phi_{L}\left(x^{+}\right)} S_{L}, \\
S_{L}=\frac{e^{-2 i \pi p}}{2 i \sin 2 \pi p} \int_{0}^{2 \pi} e^{2 \Phi_{L}}+\int_{0}^{x^{+}} e^{2 \Phi_{L}}
\end{gathered}
$$

and similarly for $v_{r}\left(x^{-}\right)$. . W We have the diagonal monodromies $S_{L}\left(x^{+}+2 \pi\right)=$ $e^{2 \pi p} S_{L}\left(x^{+}\right), \quad S_{R}\left(x^{-}+2 \pi\right)=e^{2 \pi p} S_{R}\left(x^{-}\right)$. In order to study the reduced symplectic structure, let us consider the pair of dynamical variables

$$
\begin{gathered}
\alpha_{L} \equiv j_{L}^{+}=\partial_{+} w_{L}+2 \partial_{+} \Phi_{L} w_{L}, \\
\beta_{L} \equiv v_{L}
\end{gathered}
$$

and similarly for the right-movers. The constraints $\alpha_{L}=\mu^{+}, \alpha_{R}=\mu^{-}$imply $d \alpha_{L}=d \alpha_{R}=0$. One can show, moreover, that $\Omega_{L}$ (or $\Omega_{R}$ ) only couples $\alpha_{L}$ and $\beta_{L}$ (or $\alpha_{R}$ and $\beta_{R}$ ) with each other, and not with the remaining variables $\Phi_{L}, g_{0 L}$ (or $\Phi_{R}, g_{0 R}$ ). They thus form a set of conjugate variables that

\footnotetext{
${ }^{5}$ Here $\varphi$ is the classical limit of $\alpha_{-} \Phi$ in Eq.(5).

${ }^{6}$ If we compare this with the general Liouville solution formula, $e^{\varphi}=\frac{A^{\prime}\left(x^{+}\right) B^{\prime}\left(x^{-}\right)}{\mu^{2}(A-B)^{2}}$, we see that $A=\mu^{+} S_{L}, B^{-1}=-\mu_{-} S_{R}$ and $\mu^{2}=\mu_{+} \mu_{-}$.
} 
becomes decoupled through the reduction; the $\beta_{L, R}$ are gauge variables that do not enter into $e^{\varphi}$. This means in particular that the form of the Poisson brackets between quantities containing $\alpha$ and $\Phi$ but not $\beta$ (that is, functions on the physical Liouville phase space) is unchanged by the reduction. Let us now consider the matrix elements

$$
\left(g_{L} g_{0}^{-1}\right)_{22}=w_{L} e^{\Phi_{L}},\left(g_{L} g_{0}^{-1}\right)_{21}=e^{-\Phi_{L}}
$$

which are independent of $\beta_{L}$. After reduction, we rename for convenience $\psi_{i}:=\left(g_{L}^{r e d} g_{0}^{-1}\right)_{2 i}, i=1,2$. \ $\psi_{1}$ and $\psi_{2}$ are nothing but the two solutions of the second order differential equation

$$
\psi_{i}^{\prime \prime}+T_{++} \psi_{i}=0, \quad(i=1,2)
$$

$\left(T_{++}=-\frac{1}{2}\left(\partial_{+} \varphi\right)^{2}+\partial_{+}^{2} \varphi\right.$ the Liouville energy-momentum tensor $)$ which describes the associated linear system appearing in the Lax pair approach to the theory [10], and is closely related to the uniformization equation. As the $g_{L 2 i}$ do not contain $\beta_{L}$, the Poisson brackets for the $\psi_{i}$ have the same form as those for the $\left(g_{L} g_{0}^{-1}\right)_{2 i}$ before reduction, whence?

$$
\left\{\psi_{i}\left(x^{+}\right), \psi_{i}\left(y^{+}\right)\right\}=\frac{\pi}{2 k} \epsilon\left(x^{+}-y^{+}\right) \psi_{i}\left(x^{+}\right) \psi_{i}\left(y^{+}\right)
$$

$(i=1,2)$ and

$$
\begin{gathered}
\left\{\psi_{1}\left(x^{+}\right), \psi_{2}\left(y^{+}\right)\right\}=-\frac{\pi}{2 k} \epsilon\left(x^{+}-y^{+}\right)\left[\psi_{1}\left(x^{+}\right) \psi_{2}\left(y^{+}\right)\right. \\
\left.+\frac{4}{e^{-4 \pi i p \epsilon\left(x^{+}-y^{+}\right)}-1} \psi_{2}\left(x^{+}\right) \psi_{1}\left(y^{+}\right)\right]
\end{gathered}
$$

where $\epsilon(x)$ is the step function. From the $\psi_{i} \equiv\left(g_{L}^{(r e d)} g_{0}^{-1}\right)_{2 i}$ with diagonal monodromy we can now reconstruct the general $g_{L 2 i}^{(r e d)}$, or $\psi_{i}^{g_{0}}$, by multiplying with $g_{0}: \psi_{i}^{\left(g_{0}\right)}=\psi_{j} g_{0 j i}$, and in this way we will obtain the Poisson brackets

\footnotetext{
${ }^{7}$ Observe that $A=\frac{\psi_{1}}{\psi_{2}}$ (cf. previous footnote).

${ }^{8}$ This agrees, of course, with the classical limit of the results of Gervais and Neveu for the exchange algebra of the quantized $\psi_{i}$ [11], if one takes the normalization/notation difference $\psi_{1}=\frac{1}{2 \pi i p} \mu^{+} \psi_{2}^{(\mathrm{GN})}, \psi_{2}=\psi_{1}^{(\mathrm{GN})}$ into account.
} 
of the $\psi_{i}^{g_{0}}$ on the extended phase space $\Gamma_{L}$. They are of course just given by the known Poisson brackets of the $g_{L 2 i}$ before reduction, thus

$$
\left\{\psi_{i}^{g_{0}}\left(x^{+}\right), \psi_{j}^{g_{0}}\left(y^{+}\right)\right\}=\psi_{k}^{g_{0}}\left(x^{+}\right) \psi_{l}^{g_{0}}\left(y^{+}\right)\left(r^{ \pm}\right)_{i j}^{k l}
$$

where the upper sign applies when $x^{+}>y^{+}$.

As the moment map $m=\left(\gamma^{+}, \gamma^{-}\right)$does not contain $\beta$ either, we know now that it will continue to work correctly in the reduced setup. Hence,

$$
\delta_{\epsilon} \psi_{i}^{g_{0}}=<\epsilon,\{m, \psi\} m^{-1}>
$$

is the desired transformation of $\psi_{i}^{g_{0}}$ under $\operatorname{sl}(2)$ transformations, for both $\mathrm{L}$ and $\mathrm{R}$ sectors. The map

$$
\psi_{i}^{g_{0}} \rightarrow \psi_{j}^{g_{0}} t_{j i}
$$

where $t$ is a solution of the classical limit of Eq.(4),

$$
\left\{t^{1}, t^{2}\right\}=\left[r, t^{1} t^{2}\right]
$$

( $r$ as in Eq.(11)) is Poisson-Lie, i.e. leaves the Poisson brackets Eq.(13) invariant. Remarkably, as was established in refs. [2], [3], the PL-covariant Poisson brackets Eq.(13) and their quantum counterparts Eq.(3) can also be obtained in a smaller phase space $\hat{\Gamma}$ where $g_{0 L, R}$ is not an independent dynamical variable, but has been "gauge-fixed" to become a function of $p$ :

$$
\psi_{i}^{g^{0}} \rightarrow \psi_{i}^{\hat{g}^{0}}
$$

with $\hat{g}^{0}=\hat{g}^{0}(p)$. The $\psi_{i}^{\hat{g}^{0}}$ are different from the "Bloch waves" o $\psi_{i}$ where $g_{0}=1$. If we introduce the notation $\xi_{-\frac{1}{2}}^{\left(\frac{1}{2}\right)}=\psi_{1}^{\hat{g}^{0}}$ and $\xi_{+\frac{1}{2}}^{\left(\frac{1}{2}\right)}=\psi_{2}^{\hat{g}^{0}}$, we have (classically)

$$
\left.\xi_{M}^{\left(\frac{1}{2}\right)}=\sqrt{-\frac{\pi}{2 p \sin 2 \pi p}} \sum_{j=1}^{2} \mid \frac{1}{2} p\right)_{M}^{j} \psi_{j}
$$

with

$$
\left.\left.\mid \frac{1}{2} p\right)_{M}^{1}=e^{2 i \pi M p}, \mid \frac{1}{2} p\right)_{M}^{2}=p e^{-2 i \pi M p}
$$

\footnotetext{
${ }^{9}$ This name is suggested by their diagonal monodromy, i.e. periodic behaviour up to a factor.
} 
On the quantum level, the $\xi$ fields are nothing but the $J=\frac{1}{2}$ case of the fields of section 2. They fulfill the same relations Eq.(13) (or Eq.(3) on the quantum level) as the $\psi^{g_{0}}$ even though they live on a different phase space $\hat{\Gamma}$. In fact the $\xi$ fields are functions on the physical phase space $\Gamma_{\text {phys }}$ since they have a fixed monodromy, just as the $\psi_{i}$ 's $\mathrm{s}$. Fixing the monodromy is just a way of dividing out the symmetry group $G=S L(2)$ in Eq.(2). It is clear, therefore, that there cannot exist a moment map which acts nontrivially on the $\xi$ 's while leaving invariant $\Gamma_{\text {phys }}$. However, as we will see in the next section, it is in fact possible to find a moment map that acts properly on the $\xi$ 's, while leaving at least a large subspace of $\Gamma_{\text {phys }}$ invariant.

\section{Realization of the quantum group symme- try on the physical phase space}

We will now turn to a completely new approach [12] 114 that tries to realize the quantum group symmetry on $\Gamma_{\text {phys }}$ rather than its extension $\Gamma_{L} \times \Gamma_{R}$. Our starting point is Eq.(8), so we will work directly on the quantum level.

\subsection{Definition of the action by coproduct}

The basic, and rather surprising observation is that there exists a realization of the quantum moment map $M$ in terms of the simplest $\xi$ fields themselves, namely $\xi_{ \pm \frac{1}{2}}^{\left(\frac{1}{2}\right)}$. A particular case of Eq.(3) is $\left(x_{>}>x\right) \Pi$

$$
\begin{gathered}
\xi_{-\frac{1}{2}}^{\left(\frac{1}{2}\right)}\left(x_{>}\right) \xi_{M}^{(J)}(x)=q^{M} \xi_{M}^{(J)}(x) \xi_{-\frac{1}{2}}^{\left(\frac{1}{2}\right)}\left(x_{>}\right) \\
\xi_{\frac{1}{2}}^{\left(\frac{1}{2}\right)}\left(x_{>}\right) \xi_{M}^{(J)}(x)=q^{-M} \xi_{M}^{(J)}(x) \xi_{\frac{1}{2}}^{\left(\frac{1}{2}\right)}\left(x_{>}\right)+ \\
\frac{\left(1-q^{2}\right)}{q^{\frac{1}{2}}}\left\langle J, M+1\left|J_{+}\right| J, M\right\rangle \xi_{M+1}^{(J)}(x) \xi_{-\frac{1}{2}}^{\left(\frac{1}{2}\right)}\left(x_{>}\right) .
\end{gathered}
$$

\footnotetext{
${ }^{10}$ Of course, the monodromy of the $\xi$ 's is no longer diagonal; it is upper triangular 12 .

${ }^{11}$ We drop the index + on the arguments from now on, as we will discuss only the left movers explicitly.
} 
This has exactly the form of Eq.(9) , if we identify, up to constants, $\xi_{\frac{1}{2}}^{\left(\frac{1}{2}\right)}\left(x_{>}\right)$ with $M\left(J_{+}\right)$, and $\xi_{-\frac{1}{2}}^{\left(\frac{1}{2}\right)}\left(x_{>}\right)$with $M\left(q^{J_{3}}\right)$. The crucial difference with the general transformation law is that the role of generators is played by fields that depend upon the worldsheet variable $x_{>}$. This is possible since the braiding matrix of $\xi^{\left(\frac{1}{2}\right)}\left(x_{>}\right)$with a general field $\xi_{M}^{(J)}(x)$ only depends upon the sign of $\left(x_{>}-x\right)$. Thus we may realize the Borel subalgebra $\mathcal{B}_{+}$of $U_{q}(\operatorname{sl}(2))$ simply by the $\xi^{\left(\frac{1}{2}\right)}$ fields taken at an arbitrary point (within the periodicity interval $[0,2 \pi]$ ) such that this difference is positive. Accordingly, we will write, keeping in mind the $x_{>}$dependence,

$$
\begin{aligned}
& M\left[J_{+}\right]_{x_{>}} \equiv \kappa_{+}^{>} \xi_{\frac{1}{2}}^{\left(\frac{1}{2}\right)}\left(x_{>}\right), \\
& M\left[q^{J_{3}}\right]_{x_{>}} \equiv \kappa_{3}^{>} \xi_{-\frac{1}{2}}^{\left(\frac{1}{2}\right)}\left(x_{>}\right) .
\end{aligned}
$$

In order to get agreement with Eq.(9), the normalization constants $\kappa_{+}^{>}$and $\kappa_{3}^{>}$need to fulfill $\frac{\kappa_{+}^{>}}{\kappa_{3}^{>}}=\frac{q^{\frac{1}{2}}}{1-q^{2}}$. A similar logic applies to the other Borel subalgebra $\mathcal{B}_{-}$. One defines

$$
\begin{aligned}
& M\left[J_{-}\right]_{x_{<}} \equiv \kappa_{-}^{<} \xi_{-\frac{1}{2}}^{\left(\frac{1}{2}\right)}\left(x_{<}\right), \\
& M\left[q^{J_{3}}\right]_{x_{<}} \equiv \kappa_{3}^{<} \xi_{\frac{1}{2}}^{\left(\frac{1}{2}\right)}\left(x_{<}\right),
\end{aligned}
$$

with $\frac{\kappa_{-}^{<}}{\kappa_{3}^{<}}=\frac{q^{-\frac{1}{2}}}{1-q^{-2}}$. Here $x_{<}$needs to be smaller than $x$, so that the other $R$-matrix is relevant.

One can verify now that the action of the $M\left[J_{+}\right], M\left[q^{J_{3}}\right], M\left[J_{-}\right]$generates the quantum group symmetries of the operator product and the braiding of the $\xi_{M}^{(J)}$. Let us demonstrate this for the case of the operator product. We consider the product $\xi_{M_{1}}^{\left(J_{1}\right)}\left(x_{1}\right) \xi_{M_{2}}^{\left(J_{2}\right)}\left(x_{2}\right)$ of two general $\xi$ fields. If $x_{>}>x_{1,2}$ we have, say, for $J^{a} \in \mathcal{B}_{+}$,

$$
\begin{gathered}
M\left[J^{a}\right]_{x>} \xi_{M_{1}}^{\left(J_{1}\right)}\left(x_{1}\right) \xi_{M_{2}}^{\left(J_{2}\right)}\left(x_{2}\right)=\xi_{N_{1}}^{\left(J_{1}\right)}\left(x_{1}\right) \xi_{N_{2}}^{\left(J_{2}\right)}\left(x_{2}\right) \Lambda_{b c}^{a} \times \\
\left\{\Lambda_{d e}^{b}\left[J^{d}\right]_{N_{1} M_{1}}\left[J^{e}\right]_{N_{2} M_{2}}\right\} M\left(J^{c}\right)_{x_{>}} \cdot
\end{gathered}
$$

Here $\left[J^{a}\right]_{N M}$ denotes the representation matrix of the generator $J^{a}$ in the spin $J_{1}$ representation, and $\Lambda_{b c}^{a}$ is the coefficient matrix of the coproduct. On 
the other hand, in ref. 115 the complete fusion of the $\xi$ fields was shown to be given (in the coordinates of the sphere) by

$$
\begin{gathered}
\xi_{M_{1}}^{\left(J_{1}\right)}\left(x_{1}\right) \xi_{M_{2}}^{\left(J_{2}\right)}\left(x_{2}\right)=\sum_{J_{12}=\left|J_{1}-J_{2}\right|}^{J_{1}+J_{2}} g_{J_{1} J_{2}}^{J_{12}}\left(J_{1}, M_{1} ; J_{2}, M_{2} \mid J_{12}\right) \times \\
\sum_{\{\nu\}} \xi_{M_{1}+M_{2}}^{\left(J_{12},\{\nu\}\right)}\left(x_{2}\right)\left\langle\varpi_{J_{12}},\{\nu\}\left|V_{J_{2}-J_{12}}^{\left(J_{1}\right)}\left(e^{i x_{1}}-e^{i x_{2}}\right)\right| \varpi_{J_{2}}\right\rangle,
\end{gathered}
$$

where $\left(J_{1}, M_{1} ; J_{2}, M_{2} \mid J_{12}\right)$ are the $q$-Clebsch-Gordan coefficients, and $g_{J_{1} J_{2}}^{J_{12}}$ are the so-called coupling constants, which depend on the spins only. The primary fields $V_{m}^{(J)}(z)$ whose matrix elements appear on the right-hand side are the Bloch wave operators with diagonal monodromy, which are direct generalizations of the $\psi_{i}$ fields of the previous section $\left(V_{-\frac{1}{2}}^{\left(\frac{1}{2}\right)}=\psi_{1}, V_{+\frac{1}{2}}^{\left(\frac{1}{2}\right)}=\psi_{2}\right.$.). They are linearly related to the $\xi$ fields, similarly to Eq.(14). The multiindex $\{\nu\}$ denotes descendants. If we now apply this expansion to both sides of Eq.(19), we find immediately the relation

$$
\begin{gathered}
\sum_{N_{1}+N_{2}=N_{12}}\left(J_{1}, N_{1} ; J_{2}, N_{2} \mid J_{12}\right) \Lambda_{d e}^{b}\left[J^{d}\right]_{N_{1} M_{1}}\left[J^{e}\right]_{N_{2} M_{2}}= \\
\left(J_{1}, M_{1} ; J_{2}, M_{2} \mid J_{12}\right)\left[J^{b}\right]_{N_{12} M_{12}},
\end{gathered}
$$

which is just the standard form of the recurrence relation for the $\left(\mathrm{q}^{-}\right) 3 j$ symbols. A similar analysis for the case of braiding gives

$$
\left(J_{1}, J_{2}\right)_{N_{1} N_{2}}^{P_{2} \Lambda_{d e}} \Lambda_{d e}^{b}\left[J^{d}\right]_{N_{1} M_{1}}\left[J^{e}\right]_{N_{2} M_{2}}=\Lambda_{d e}^{b}\left[J^{d}\right]_{P_{2} N_{2}}\left[J^{e}\right]_{P_{1} N_{1}}\left(J_{1}, J_{2}\right)_{M_{1} M_{2}}^{N_{2} N_{1}}
$$

which is just the condition that the universal $R$ matrix interchanges the two coproducts. The same relations follow from a consideration of the $M$ operators corresponding to $\mathcal{B}_{-}$. Thus, the $\xi_{M}^{(J)}$ generate themselves the symmetries of their operator algebra in a kind of bootstrap fashion.

\subsection{The algebra of the $M$ operators}

So far we have just considered the action of the $M\left[J^{a}\right]$ on the $\xi_{M}^{(J)}$ but not their commutation relations with each other. If $M$ is a homomorphism as assumed below Eq.(8), they should of course just reproduce the $U_{q}(s l(2))$ algebra. It 
is in fact known - and we will see below - that the most general commutation relations compatible with the $U_{q}(s l(2))$ coproduct which appears in Eq.(9) are just those obtained from the standard ones by certain linear redefinitions of the generators. It will turn out that the $M$ operators should be viewed as homomorphic to such redefined generators, and not to the standard ones. Let us explain this in some more detail. We will ignore at first the complications introduced by the position dependence of the $M$ operators, and make up for this later. The most general commutation relation for $M\left[J_{+}\right]$and $M\left[q^{J_{3}}\right]$ compatible with Eq.(9) is

$$
q M\left[J_{+}\right] M\left[q^{J_{3}}\right]-M\left[q^{J_{3}}\right] M\left[J_{+}\right]=C_{+}
$$

where $C_{+}$is a central term which commutes with all the $\xi$ 's. Similarly, one has

$$
M\left[q^{J_{3}}\right] M\left[J_{-}\right]-q^{-1} M\left[J_{-}\right] M\left[q^{J_{3}}\right]=C_{-} .
$$

Finally, by considering the action of $\left[M\left[J_{+}\right], M\left[J_{-}\right]\right]$on $\xi_{M}^{(J)}$, one obtains

$$
\begin{aligned}
& {\left[M\left[J_{+}\right], M\left[J_{-}\right]\right]=\frac{\left.\left(M\left[q^{J_{3}}\right]\right)^{2}-\left(M\left[q^{-J_{3}}\right]\right)^{2}\right)}{q-q^{-1}}+} \\
& \left(C_{3} M\left[q^{-J_{3}}\right]+C_{+} M\left[J_{-}\right]+C_{-} M\left[J_{+}\right]\right) M\left[q^{-J_{3}}\right]
\end{aligned}
$$

where $C_{3}$ is a third central term $[\mathbf{T}$. One can bring these commutation relations into the standard form $\left[J_{+}^{\prime}, J_{-}^{\prime}\right]=\left\lfloor 2 J_{3}^{\prime}\right\rfloor, J_{ \pm}^{\prime} q^{J_{3}^{\prime}}=q^{\mp 1} q^{J_{3}^{\prime}} J_{ \pm}^{\prime}$ by means of the redefinitions

$$
J_{ \pm}^{\prime}:=\rho\left(M\left[J_{ \pm}\right] \pm \frac{C_{ \pm}}{1-q^{ \pm 1}} M\left[q^{-J_{3}}\right]\right), \quad q^{ \pm J_{3}^{\prime}}:=\rho^{ \pm 1} M\left[q^{ \pm J_{3}}\right]
$$

with

$$
\rho^{-4}=\left(q-q^{-1}\right)\left(C_{+} C_{-} \frac{1+q}{1-q}-C_{3}\right)+1
$$

However, in the present field theoretic realization $\rho^{-4}$ will be given in terms of a bilinear in $\xi^{\left(\frac{1}{2}\right)}$ fields, and $\rho=\left(\rho^{-4}\right)^{-1 / 4}$ possesses no clear meaning. Therefore the above redefinition is formal, and we are forced to stick with the

\footnotetext{
${ }^{12}$ Of course $C_{ \pm}, C_{3}$ are not central extensions of $U_{q}(\operatorname{sl}(2))$ (which don't exist!).
} 
somewhat nonstandard realization of the $U_{q}(s l(2))$ commutation relations. Let us now consider the concrete realization of the $M$ operators in terms of the $\xi^{\left(\frac{1}{2}\right)}$ fields, which introduces a position dependence. In this situation, we have to define what we mean exactly when we talk about commutation relations of the $M$ operators. For instance, in the case of $\mathcal{B}_{+}$one finds

$$
q M\left[J_{+}\right]_{x_{>}} M\left[q^{J_{3}}\right]_{x_{>}^{\prime}}-M\left[q^{J_{3}}\right]_{x_{>}} M\left[J_{+}\right]_{x_{>}^{\prime}}=C_{+}\left(x_{>}, x_{>}^{\prime}\right)
$$

with $x_{>}>x_{>}^{\prime}$. Notice that the positions $x_{>}, x_{>}^{\prime}$ are not interchanged as the ordering must be respected also when the $M$ operators act on themselves. We call this definition of the commutation relations fixed point (FP) commutator. A similar convention applies to the case of $\mathcal{B}_{-}$. Explicitly, one computes

$$
\begin{aligned}
& C_{+}\left(x_{>}, x_{>}^{\prime}\right)=\sqrt{q} \kappa_{+}^{>} \kappa_{3}^{>} \xi_{0}^{\left[\frac{1}{2}, \frac{1}{2}\right](0)}\left(x_{>}, x_{>}^{\prime}\right), \\
& C_{-}\left(x_{<}, x_{<}^{\prime}\right)=\frac{1}{\sqrt{q}} \kappa_{-}^{<} \kappa_{3}^{<} \xi_{0}^{\left[\frac{1}{2}, \frac{1}{2}\right](0)}\left(x_{<}, x_{<}^{\prime}\right),
\end{aligned}
$$

where $\xi_{0}^{\left[\frac{1}{2}, \frac{1}{2}\right](0)}\left(x_{>}, x_{>}^{\prime}\right)$ is simply the singlet $\left(\frac{1}{2}, M ; \frac{1}{2},-M \mid 0\right) \xi_{M}^{\left(\frac{1}{2}\right)}\left(x_{>}\right) \xi_{-M}^{\left(\frac{1}{2}\right)}\left(x_{>}^{\prime}\right)$. The reason that $C_{+}$is central with respect to any field $\xi_{M}^{(J)}(x)$ with $x<x_{>}^{\prime}<$ $x_{>}$, and similarly for $C_{-}$, is simply that the $R$-matrix $(J, 0)_{M 0}^{0 N}$ is trivial.

It remains to define commutation relations between elements of $\mathcal{B}_{+}$and $\mathcal{B}_{\text {-. }}$. Here we meet the obstacle that apparently, we cannot hold fixed the positions in the interchange as required by the FP prescription, because operators from $\mathcal{B}_{+}$are not defined at points $x_{<}$, and similarly for $\mathcal{B}_{-}$at $x_{>}$. Fortunately, the monodromy operation comes to our rescue here. As already mentioned, the $\xi_{M}^{(J)}$ fields are linearly related to the Bloch wave operators with diagonal monodromy (cf. section 5) [3]. From this, it is straightforward to deduce

$$
\begin{aligned}
& \xi_{-\frac{1}{2}}^{\left(\frac{1}{2}\right)}(x+2 \pi)=\xi_{\frac{1}{2}}^{\left(\frac{1}{2}\right)}(x), \\
& \xi_{\frac{1}{2}}^{\left(\frac{1}{2}\right)}(x+2 \pi)=2 \sqrt{q} \cos (2 \pi p) \xi_{\frac{1}{2}}^{\left(\frac{1}{2}\right)}(x)-q \xi_{-\frac{1}{2}}^{\left(\frac{1}{2}\right)}(x)
\end{aligned}
$$

\footnotetext{
${ }^{13}$ We do not give the quantum equivalents of the coefficients $\left.\mid \frac{1}{2} p\right)_{M}^{j}$ of Eq.(14), but they are known (cf. ref. [3]).
} 
and the inverse relation

$$
\begin{aligned}
& \xi_{-\frac{1}{2}}^{\left(\frac{1}{2}\right)}(x-2 \pi)=\frac{2}{\sqrt{q}} \cos (2 \pi p) \xi_{-\frac{1}{2}}^{\left(\frac{1}{2}\right)}(x)-\frac{1}{q} \xi_{\frac{1}{2}}^{\left(\frac{1}{2}\right)}(x) \\
& \xi_{\frac{1}{2}}^{\left(\frac{1}{2}\right)}(x-2 \pi)=\xi_{-\frac{1}{2}}^{\left(\frac{1}{2}\right)}(x) .
\end{aligned}
$$

Note that $p$ is an operator which does not commute with the $\xi_{M}^{(J)}$.

The expression Eq.(3) for the case $x<x^{\prime}$ is valid for $x^{\prime}-x \in[0,2 \pi]$. Thus if we start from $M\left[J_{-}\right]_{x_{<}} \xi_{M}^{(J)}(x)$ with $x \in[0,2 \pi], x_{<}<0, x-x_{<} \in[0,2 \pi]$, we can reexpress $M\left[J_{-}\right]_{x_{<}}$in terms of an operator at $x_{>}:=x_{<}+2 \pi \in[0,2 \pi]$. Thus we can define

$$
M\left[J_{-}\right]_{x_{>}}:=\kappa_{-}^{>} \xi_{-\frac{1}{2}}^{\left(\frac{1}{2}\right)}\left(x_{>}-2 \pi\right)=\kappa_{-}^{>}\left(\frac{2}{\sqrt{q}} \cos (2 \pi p) \xi_{-\frac{1}{2}}^{\left(\frac{1}{2}\right)}\left(x_{>}\right)-\frac{1}{q} \xi_{\frac{1}{2}}^{\left(\frac{1}{2}\right)}\left(x_{>}\right)\right)
$$

with

$$
\frac{\kappa_{-}^{>}}{\kappa_{3}^{>}}=\frac{q^{-\frac{1}{2}}}{1-q^{-2}}
$$

We can then also identify the Cartan generators at $x_{<}$and $x_{>}, M\left[q^{J_{3}}\right]_{x_{<}} \equiv$ $\kappa_{3}^{<} \xi_{\frac{1}{2}}^{\left(\frac{1}{2}\right)}\left(x_{<}\right)=M\left[q^{J_{3}}\right]_{x>} \equiv \kappa_{3}^{>} \xi_{-\frac{1}{2}}^{\left(\frac{1}{2}\right)}\left(x_{>}\right)$, using again Eqs. 29). The explicit expressions for the monodromy of $\xi_{ \pm \frac{1}{2}}^{\left(\frac{1}{2}\right)}(\sigma)$ lead to the following relation between $M\left[J_{ \pm}\right], M\left[q^{J_{3}}\right]$ and $\cos (2 \pi p)$ :

$$
2 \cos (2 \pi p) M\left[q^{J_{3}}\right]_{x_{>}}=\left(q-q^{-1}\right)\left(M\left[J_{-}\right]_{x_{>}}-M\left[J_{+}\right]_{x_{>}}\right) .
$$

This shows that the zero mode $p$ should be viewed as an element of the universal enveloping algebra. With the definition of $M\left[J_{-}\right]_{x^{-}}$above, we can now write the F.P. commutation relations for $M\left[J_{+}\right]$and $M\left[J_{-}\right]$:

$$
M\left[J_{+}\right]_{x_{>}} M\left[J_{-}\right]_{x_{>}^{\prime}}-M\left[J_{-}\right]_{x_{>}} M\left[J_{+}\right]_{x_{>}^{\prime}}=M[D]_{x_{>}, x_{>}^{\prime}}+\frac{M\left[q^{J_{3}}\right]_{x_{>}} M\left[q^{J_{3}}\right]_{x_{>}^{\prime}}}{q-q^{-1}} .
$$

Here $M[D]$ is defined by

$$
M[D]_{x_{>}, x_{>}^{\prime}}=\frac{1}{q-q^{-1}} 2 \cos (2 \pi p) C_{+}\left(x_{>}, x_{>}^{\prime}\right) .
$$


$M[D]$ is a realization of the operator $\left(C_{+} M\left[J_{-}\right]+C_{-} M\left[J_{+}\right]\right) M\left[q^{-J_{3}}\right]$ in Eq.(25), and we have

$$
C_{3}=\frac{1}{q-q^{-1}}, \quad C_{+}=-C_{-}
$$

so that there is no term proportional to $M\left[q^{-J_{3}}\right]^{2}$ in Eq. 225). Note that the operator $M\left[q^{-J_{3}}\right]$ is not realized by any combination of the $\xi^{\left(\frac{1}{2}\right)}$ and $p$. If Rather, it is only the particular combination above which can be constructed. However, the algebra of $M\left[J_{ \pm}\right], M\left[q^{J_{3}}\right], M[D]$ is equivalent to that of $M\left[J_{ \pm}\right], M\left[q^{J_{3}}\right], M\left[q^{-J_{3}}\right]$, and commutations of $M[D]$ with the other operators can always be reexpressed in terms of $M\left[J_{ \pm}\right], M\left[q^{J_{3}}\right], M[D]$ again. Thus we obtain a consistent formulation of the algebra in terms of our F.P. prescription.

\subsection{Invariance of the physical phase space}

We now discuss the action of the $M$ operators on the physical phase space, as announced at the end of section 4. The interesting observables, or functions on the physical phase space, are given by the Liouville exponentials of Eq.(5). The canonical variables $\Phi$ and $\Pi$ can be reconstructed from them by taking suitable derivatives [6]. Thus the $e^{-J \alpha_{-} \Phi}\left(x^{+}, x^{-}\right)\left(x^{+}, x^{-}\right.$varying) can be viewed as probes of different regions of $\Gamma_{\text {phys }}$. According to the remark at the end of section 3, we expect the $M$ operators for left and right-moving sectors together to be given by

$$
M\left[J_{ \pm}\right]_{x_{>}^{+}, x_{>}^{-}}^{(L R)}=M\left[J_{ \pm}\right]_{x_{>}^{+}} \bar{M}\left[\bar{q}^{\bar{J}_{3}}\right]_{x_{>}^{-}}+M\left[q^{J_{3}}\right]_{x_{>}^{+}} \bar{M}\left[\bar{J}_{ \pm}\right]_{x_{>}^{-}}
$$

and

$$
M\left[q^{J_{3}}\right]_{x_{>}^{+}, x_{>}^{-}}^{(L R)}=M\left[q^{J_{3}}\right]_{x_{>}^{+}} \bar{M}\left[\bar{q}^{-\bar{J}_{3}}\right]_{x_{>}^{-}}
$$

\footnotetext{
${ }^{14}$ However, it can be realized by $\xi_{\frac{1}{2}}^{\left(-\frac{1}{2}\right)}$, see ref. [12]. One can use this representation to verify that Eq.(33) is obtained by defining $M\left[J_{ \pm}\right] M\left[q^{-J_{3}}\right]$ via a renormalized shortdistance product.
} 
where the $\bar{M}$ operators are defined exactly like the $M$ operators, but in terms of the $\bar{\xi}_{M}^{(J)}$ fields, and $\bar{q} \equiv q^{-1}$.'丁 Inserting the definitions, one finds

$$
\begin{gathered}
M\left[J_{+}\right]_{x_{>}^{+}, x_{>}^{-}}^{(L R)}=e^{-\frac{1}{2} \alpha_{-} \Phi}\left(x_{>}^{+}, x_{>}^{-}\right) \\
M\left[J_{-}\right]_{x_{<}^{+}, x_{<}^{-}}^{(L R)}=e^{-\frac{1}{2} \alpha_{-} \Phi}\left(x_{<}^{+}, x_{<}^{-}\right) \\
M\left[q^{J_{3}}\right]_{x_{>}^{+}, x_{>}^{-}}^{(L R)}=\xi_{-\frac{1}{2}}^{\left(\frac{1}{2}\right)}\left(x_{>}^{+}\right) \bar{\xi}_{-\frac{1}{2}}^{\left(\frac{1}{2}\right)}\left(x_{>}^{-}\right)
\end{gathered}
$$

up to irrelevant constants. Thus, the action of the lowering/raising operators is just given by the simplest Liouville exponential itself! Invariance under the action of, say, $J_{+}$thus just means that $e^{-\frac{1}{2} \alpha_{-} \Phi}\left(x_{>}^{+}, x_{>}^{-}\right)$and $e^{-J \alpha_{-} \Phi}\left(x^{+}, x^{-}\right)$ are relatively local. This is certainly true for spacelike separations, hence in particular if the conditions $x_{>}^{+}>x^{+}, x_{>}^{-}>x^{-}$are fulfilled $\square$. On the other hand, if (exactly) one of the conditions is violated, the two exponentials are not mutually local and thus $e^{-J \alpha_{-} \Phi}\left(x^{+}, x^{-}\right)$is not invariant under the action of the $M\left[J_{ \pm}\right]^{(L R)}$ operator. Now clearly we can move the window of "allowed" $x^{ \pm}$values anywhere we like by changing $x_{>}^{+}, x_{>}^{-}$appropriately, using the monodromy operation if necessary. Thus, the present formulation of the quantum group generators allows to consider the subspaces of $\Gamma_{\text {phys }}$ given by local functionals in a $2 \pi$ neighborhood of the values $x_{>}^{+}, x_{>}^{-}$, which in turn can be chosen arbitrarily. In other words, one has movable windows on the physical phase space which are left invariant by the $M$ operators.

\section{Conclusions}

We have presented two rather different approaches to the problem of constructing moment maps - or q-Noether charges - for the quantum group symmetry in conformal field theory. The first one uses the standard apparatus of Poisson Lie symmetry, and we employed Hamiltonian reduction to

\footnotetext{
${ }^{15}$ In fact, in order to realize the operator $\bar{M}\left[\bar{q}^{-\bar{J}_{3}}\right]_{x_{>}^{-}}$one needs to use the field $\bar{\xi}_{+\frac{1}{2}}^{\left(-\frac{1}{2}\right)}$ (cf. last footnote).

16 (or in fact, if both of them are violated; but this case is equivalent to the previous one because of the periodicity of the Liouville exponentials, which allows to apply a double monodromy operation to both $\xi_{ \pm \frac{1}{2}}^{\left(\frac{1}{2}\right)}\left(x^{+}\right)$and $\bar{\xi}_{ \pm \frac{1}{2}}^{\left(\frac{1}{2}\right)}\left(x^{-}\right)$, leading back to the previous case.)
} 
obtain the moment map for Liouville theory from the known result for the WZW case. Our analysis was obviously incomplete as we presented only the classical moment map. However, it is conceptually clear how to extend the analysis to the quantum case and one expects no major technical obstacles; work on this is in progress [13]. Furthermore, an extension to general Toda theories seems both desirable and feasible.

On the other hand, in the "intrinsic" approach working on the physical phase space we were able to obtain a realization directly on the quantum level. However, this approach, which starts from a "phenomenological" observation on the structure of the $R$ matrix, is conceptually still much less understood. Its most striking feature is perhaps the position dependence of the generators, which suggests an embedding into a Kac-Moody type structure, though of course Liouville theory a priori just has a global $S L(2)$ symmetry. It is intriguing that in this way one can realize a kind of BRST transformations on a gauge-fixed theory without extending the phase space. Clearly, it is important to understand better the deeper meaning of this new "weak" symmetry of the physical phase space, which leaves freely movable parts of it invariant but never all of it. We remark that the construction can be extended to $U_{q}(s l(2)) \otimes U_{\hat{q}}(s l(2))$ [12], where $\hat{q}$ is the deformation parameter which corresponds to the second screening charge $\alpha_{+}$. This symmetry is well known within the description of minimal models [16], where only half-integer positive spins are relevant, but much less in the context of arbitrary spins as required for Liouville theory.

In ref. [14], it was shown how the second approach can also be formulated directly in the Bloch wave $(\psi)$ basis, which is closely related to the standard Coulomb gas picture of conformal field theories. Remarkably, in this realization one finds a hidden $U_{q}(s l(2)) \times U_{q}(s l(2))$ symmetry $\square$ with a nonstandard coproduct structure and an additional constraint, which describes the symmetries of the Bloch wave operator algebra in the same way as the $M$ operators did for the $\xi_{M}^{(J)}$ fields.

Acknowledgements: The work contained in section 5 has been done in collaboration with C. Klimcik, while the results in section 6 were obtained together with E. Cremmer and J.-L. Gervais. I would like to thank all of them for a fruitful collaboration, and B. Jurco and A. Alekseev for many

\footnotetext{
${ }^{17}$ already in the case of one screening charge!
} 
helpful discussions.

\section{References}

[1] A. Alekseev, F. Faddeev, M. Semenov-Tian-Shanskii, A. Volkov, CERNTH-5981-91, Jan. 1991. K. Gawedzki, "Quantum Group Symmetries in Conformal Field Theory", Kyoto 1992 proceedings, Quantum and Noncommutative Analysis, p. 239; hep-th/9210100.

[2] O. Babelon, Phys. Lett. B215 (1988) 523.

[3] J.-L. Gervais, Comm. Math. Phys. 130 (1990) 257.

[4] L. Faddeev, N. Reshetikhin, L. Takhtadzhian, "Quantization of Lie Groups and Lie Algebras", LOMI-E-87, 1987.

[5] F. Falceto, K. Gawedzki, lectures at the XXVIIIth Karpacz winter school, Feb. 1992; J. Geom. Phys. 11 (1993) 251.

[6] J.-L. Gervais, J. Schnittger, Nucl. Phys. B431 (1994) 273.

[7] J.-L. Gervais, Nucl. Phys. B391 (1993) 287.

[8] A. Alekseev, I. Todorov, Nucl. Phys. B421 (1993) 413.

[9] J. Balog, L. Feher, P. Forgacs, L. O'Raifeartaigh, A. Wipf, pl B227, 1989, 214. M. Bershadsky, Comm. Math. Phys. 139 (1991) 71.

[10] O. Babelon, "From Integrable to Conformal Field Theory", Lectures given at 20th Int. GIFT seminar, Jaca, Spain, June 1989; GIFT seminar 1989, p.1 and PAR-LPTHE-89-29, June 1989.

[11] J.-L. Gervais, A. Neveu, Nucl. Phys. B238 (1984) 125.

[12] E. Cremmer, J.-L. Gervais, J. Schnittger, Comm. Math. Phys. 178 (1996) 147.

[13] C. Klimcik, J. Schnittger, work in progress. 
[14] E. Cremmer, J.-L. Gervais, J. Schnittger, "Hidden $U_{q}(s l(2)) \otimes$ $U_{q}(s l(2))$ Quantum Group Symmetry in Two-Dimensional Gravity", hep-th/9604131.

[15] E. Cremmer, J.-L. Gervais, J.-F. Roussel, Comm. Math. Phys. 161 (1994) 597.

[16] L. Alvarez-Gaume, C. Gomez, G. Sierra, Nucl. Phys. B319 (1989) 155.

[17] O. Babelon, D. Bernard, Comm. Math. Phys. 149 (1992) 279; Phys. Lett. B260 (1991) 81. 